Check for updates

Cite this: RSC Adv., 2017, 7, 25276

\title{
Observing different modes of mobility in lithium titanate spinel by nuclear magnetic resonance
}

\author{
Magnus F. Graf, (D) *a Hermann Tempel, (D) a Simone S. Köcher, (D) ab

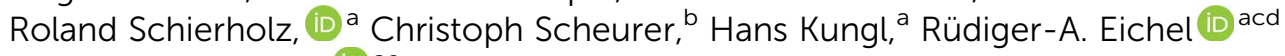 \\ and Josef Granwehr (DD ae
}

\begin{abstract}
Lithium titanate (LTO) is a spinel material that is able to reversibly intercalate Li ions with minimal changes of the unit cell dimensions ("zero-strain"), making it an attractive choice as anode material for Li ion batteries. However, the nature of the Li transport in this material is still not fully understood. Here, the Li mobility in $\mathrm{Li}_{4+x} \mathrm{Ti}_{5} \mathrm{O}_{12}$ with $x=0$ and $x \approx 1.6$ is investigated. By regularized inversion of nuclear magnetic resonance (NMR) relaxation and spin alignment echo (SAE) data and supported by DFT simulations, solid-state NMR spectra were analyzed as a function of the respective relaxation times and correlation time constants. A clear correlation between mobility and NMR spectral features was observed, suggesting the presence of local domains with high Li ion mobility. The long-range mobility is limited by the much slower hopping between such domains and appears to be faster for either larger or less ordered local domains. For $x \approx$ 1.6, spectral features indicate the formation of separate stoichiometric and overlithiated phases rather than a solid solution, yet no segregation into a fast and a slow component was observed in the relaxation and in the SAE dimension, which points towards an entangling of the two phases on a microscopic scale.
\end{abstract}

Received 8th February 2017

Accepted 30th April 2017

DOI: 10.1039/c7ra01622k

rsc.li/rsc-advances chemical overlithiation was reported to form an inserted spinel structure, ${ }^{17,18}$ with chemically inserted $\mathrm{Li}$ ions found to be trapped in $48 \mathrm{f}$ sites. Furthermore, different site occupation ratios for Li have been reported for different synthesis routes of LTO and overlithiated LTO (e.g. ref. 19-22).

For rational materials design the understanding of dynamical and electrochemical processes taking place in LTO is of utmost importance to establish structure-property relationships by complementing the static structural picture with dynamic information. Initial models of dynamic processes were made based on structural changes. ${ }^{9}$ Li diffusion in LTO depends on the size of LTO particles, with evidence for kinetic ${ }^{23}$ as well as surface equilibrium causes. ${ }^{24} \mathrm{Li}$ diffusion has been studied by neutron diffraction, ${ }^{23,25}$ impedance spectroscopy ${ }^{18}$ and chronoamperometry. ${ }^{26}$ The "ultra slow" regime was analyzed via spin alignment echo (SAE) nuclear magnetic resonance (NMR), where changes of the electric field gradient (EFG) seen by a nucleus via its quadrupolar coupling constant $C_{\mathrm{Q}}$ are probed. ${ }^{27}$ The echo decay is characterized by a correlation time constant $\tau_{\mathrm{C}}$ that may be identified as a hopping time between sites, provided that the origin and the destination of a jump are not electrically equivalent. In LTO all jumps occur between inequivalent sites with different EFGs and are therefore contributing to the SAE decay. ${ }^{12}$ Furthermore, due to the random occupancy of $16 \mathrm{~d}$ sites by Ti and Li ions (Fig. 1), even nominally identical sites show a distribution of EFG values. The complete intensity decay curve as a function of mixing time tm in an SAE experiment is usually described by one or the sum of
Jülich, Germany. E-mail: j.granwehr@fz-juelich.de

${ }^{b}$ Lehrstuhl für Theoretische Chemie, Technische Universität München, Garching, Germany

${ }^{c} R W T H$ Aachen University, Institute of Physical Chemistry, Aachen, Germany ${ }^{d} J$ ülich Aachen Research Alliance (JARA), Section JARA-Energy, Aachen, Germany

${ }^{e} R W T H$ Aachen University, Institute of Technical and Macromolecular Chemistry, Aachen, Germany 


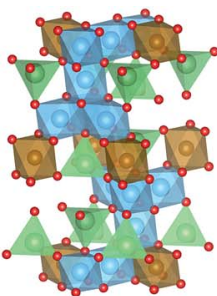

a

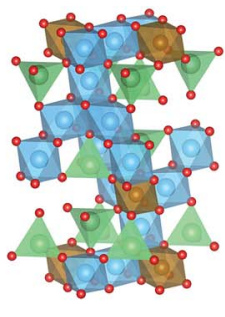

b

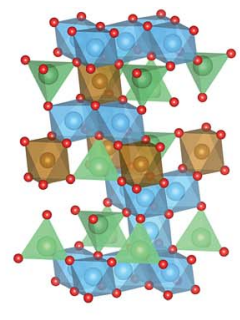

C
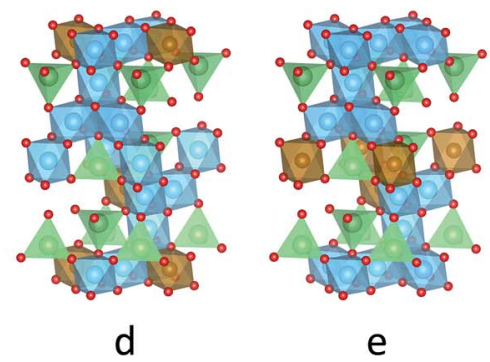

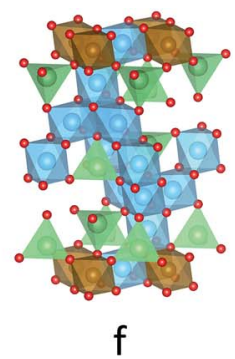

Fig. 1 Six symmetry-inequivalent stoichiometric $\mathrm{Li}_{8} \mathrm{Ti}_{10} \mathrm{O}_{24}$ unit cells (idealized space group $R 3 \mathrm{~m}$ ) used for ab initio calculation of quadrupolar coupling constants. The oxygen $\left(\mathrm{O}^{2-}\right)$ lattice is shown in red, blue octahedrons denote titanium $\left(\mathrm{Ti}^{4+}\right)$ in $16 \mathrm{~d}$ sites, brown octahedrons and green tetrahedrons represent lithium $\left(\mathrm{Li}^{+}\right)$in $16 \mathrm{~d}$ and $8 \mathrm{a}$ sites, respectively.

several stretched exponential functions. The stretched nature of these functions is generally believed to result from a distribution of time constants with an underlying exponential kernel. ${ }^{28}$

Temperature dependent measurements of conductivity ${ }^{29}$ or NMR relaxation times ${ }^{12}$ were used to obtain activation energies $E_{\mathrm{a}}$ for jumps of Li ions. So far the mechanism of Li conductivity could not be fully resolved. ${ }^{30,31}$ One reason was provided by a recent theoretical study where it was concluded that jump rates are not primarily limited by activation energies, but a combination of $E_{\mathrm{a}}$ and the availability of jump targets. ${ }^{32}$ Thus, $E_{\mathrm{a}}$ derived in temperature dependent experiments are merely effective parameters and cannot generally be interpreted as actual physical barriers of ion motion.

Inverting relaxation and diffusion data using an exponential kernel instead of fitting it with a particular model has become a routine tool in NMR. ${ }^{33-35}$ However, the common regularization with a non-negativity constraint is not physically justified once exchange processes become relevant for the dynamics of a spin system. ${ }^{36-40}$ Amorphous and multisite crystalline solid-state systems are affected in particular due to their highly branched interaction network,$^{28}$ which requires a different approach for data inversion. One suggestion was to analyze the data in the time domain, without actually inverting it. ${ }^{40} \mathrm{Here}$, the applicability of uniform penalty (UP) regularization in combination with a penalty to prevent unnecessary oscillations ${ }^{41}$ is demonstrated for the inversion of solid-state NMR data. The suitability of the algorithm for averaging in a non-inverted dimension is shown, facilitating spectral separation of the static NMR spectrum with respect to the SAE time constant. Using this technique and supported by $a b$ initio density functional theory (DFT) calculations, the dynamics of stoichiometric and chemically overlithiated LTO is investigated.

\section{Methods}

\subsection{Sample preparation and characterization}

Stoichiometric $\mathrm{Li}_{4} \mathrm{Ti}_{5} \mathrm{O}_{12}$ was purchased from Sigma-Aldrich (>99\%). $\mathrm{Li}_{4+x} \mathrm{Ti}_{5} \mathrm{O}_{12}$ with $x \approx 1.6$ was prepared by dispersing $10 \mathrm{mmol}$ of the commercial $\mathrm{Li}_{4} \mathrm{Ti}_{5} \mathrm{O}_{12}$ in $30 \mathrm{ml}$ hexane with subsequent slow addition of $2 \mathrm{ml}$ of $11 \mathrm{M}$ butyllithium (3 $\mathrm{mmol}$ ). The color of the dispersion changed immediately from white to dark blue when adding the butyllithium. The dispersion was stirred for at least 24 hours, dried under vacuum, washed three times with fresh hexane and then dried again. Complete transition of the Li from the butyllithium to the lithium titanate results in formation of $\mathrm{Li}_{4+x} \mathrm{Ti}_{5} \mathrm{O}_{12}$ with $x \approx 1.6$. All sample preparation steps were done in a glove box under argon. A sample with a lower degree of lithiation $(x \approx 0.2)$ was prepared in the same way, using $0.4 \mathrm{mmol}$ butyllithium.

X-ray diffraction was measured in transmission mode with $\mathrm{Cu}$ K-alpha radiation $(40 \mathrm{kV}, 40 \mathrm{~mA})$ with an Empyrean diffractometer (Panalytical, Netherlands) with the powder placed between two mylar foils. Microscopy was performed with a CM 20 transmission electron microscope (Philips, Netherlands) operated at $200 \mathrm{kV}$. In the glovebox the powder was placed on lacey carbon grids which were sealed in aluminium foil for transport. The bags were opened, the grids were placed in the holder and the holder was inserted into the TEM to minimize the exposure of the samples to air (max. 1 minute). PowderCell (Bundesanstalt für Materialforschung und prüfung $)^{42}$ was used to simulate diffraction patterns for stoichiometric LTO (ICSD 160655) and fully overlithiated LTO ( $x=$ 3, ICSD 160655 but Li on 16c).

\subsection{NMR spectroscopy}

All NMR measurements were recorded on a Bruker AVANCE III HD 400 spectrometer at a ${ }^{7} \mathrm{Li}$ frequency of $\omega_{0} / 2 \pi=155.5 \mathrm{MHz}$.

SAE NMR employs the Jeener-Broekaert pulse sequence $\beta_{1}-$ $t_{\mathrm{p}}-\beta_{2}-t_{\mathrm{m}}-\beta_{3}-t_{\mathrm{d}}$, where $\beta$ represents radio frequency pulses, $t_{\mathrm{p}}$ is the evolution time, $t_{\mathrm{m}}$ the mixing time and $t_{\mathrm{d}}$ the detection transient. ${ }^{43}$ It results in an echo with an intensity relative to the correlation between the quadrupolar precession frequency $\omega_{\mathrm{Q}}$ during the evolution time and during the detection transient at time $t_{\mathrm{m}}$ later. A correlation function can be mapped directly by repeating this sequence with variable $t_{\mathrm{m}}$ if the correlation time constant, $\tau_{\mathrm{C}}$, is shorter than the spin-lattice relaxation with time constant $T_{1}$, the decay of quadrupolar order with time constant $T_{1 \mathrm{Q}}$, and temperature-independent spin diffusion processes. ${ }^{27}$ SAE NMR measurements of stoichiometric and overlithiated LTO were recorded at different temperatures between $10{ }^{\circ} \mathrm{C}$ and $45{ }^{\circ} \mathrm{C}$ using a 32 step phase cycle $^{44}$ and a fixed evolution time $t_{\mathrm{p}}$ of $10 \mu \mathrm{s}$. A saturation pulse train and a suitable delay time were used between measurements to ensure consistent initial conditions. In all the experiments with a static sample, $90^{\circ}$-pulse lengths were around 6.5 $\mu \mathrm{s}$. The sample temperature was controlled by a Bruker BCU-20 
cooling unit and was recorded using a Bruker B-SVT system. The temperature was stable within $\pm 1 \mathrm{~K}$. The reported temperatures are averaged values over the experiment time.

$T_{1}$ of the overlithiated samples was measured spectrally resolved using the saturation recovery (SR) experiment with magic angle sample spinning (MAS). For this experiment the $90^{\circ}$-pulse duration was $1.1 \mu \mathrm{s}$ and the $1.3 \mathrm{~mm}$ rotor was set to a speed of $65 \mathrm{kHz}$. The sample temperature was controlled with a flow of heated nitrogen.

\subsection{NMR data analysis}

A distribution of $\tau_{\mathrm{C}}$ from SAE experiments and of $T_{1}$ from SR experiments is obtained using regularized inversion with UP regularization in combination with a penalty for zero crossing of the density function to prevent it from exhibiting unnecessary oscillations by minimizing the number of sign changes. At the same time, neither sign of the density function amplitude is preferred over the other. ${ }^{41}$

The spectral NMR information contained in the $\mathrm{SAE}^{13}$ and in the SR experiments are obtained by Fourier transformation of the transient echo signal and the free induction decay, respectively. Since a spectral density in the inverted dimension is determined from the echo decay as a function of $t_{\mathrm{m}}$ rather than fitting a particular $\tau_{\mathrm{C}}$ or $T_{1}$ to the data, the NMR spectrum corresponding to different $\tau_{\mathrm{C}}$ and $T_{1}$ can be obtained in a second dimension that is contained in the transiently recorded data already. This facilitates the independent mobility characterization of ions in different lattice sites or with different local environments whose spectrum and correlation time both vary considerably. Although the data is inverted in one dimension only, the full two-dimensional data set for $T_{1}$ and the full three-dimensional data set for $\tau_{\mathrm{C}}$ are used when performing the regularized inversion. In the spectral and in the (inverse) temperature dimension, a unity kernel is employed, hence regularization acts as a smoothing filter along the non-inverted dimensions. The advantage compared to the application of smoothing after inversion of independent one-dimensional data sets is that an algorithm acting on multi-dimensional data leads to potentially narrower features along the inverted dimension since the sensitivity-enhancing smoothing is performed simultaneously with data inversion. ${ }^{45}$

The regularization parameters for the inversion have been chosen as detailed in ref. 41 .

\subsection{Computational details of DFT simulations}

To approximate the occupational disorder of $\mathrm{Li}_{4} \mathrm{Ti}_{5} \mathrm{O}_{12}$, the stoichiometric representation $\mathrm{Li}_{8} \mathrm{Ti}_{10} \mathrm{O}_{24}$ (space group $R \overline{3} m$ ) was studied. All possible occupations of the unit cell reduce to six symmetry-inequivalent structures similar in total energy, shown in Fig. 1. The geometry of the six unit cells was optimized with CASTEP 7.01 (ref. 46) using the default ultra-soft, on-the-flygenerated pseudo-potentials and the PBE exchange-correlation functional. The forces were converged to $3 \mathrm{meV}$ with a cutoff energy of $600 \mathrm{eV}$ and a $k$-point grid of $4 \times 4 \times 2$.

The quadrupolar coupling constants $C_{\mathrm{Q}}$ for the optimized geometries were calculated with CASTEP 8.0, ${ }^{46}$ the default ultra- soft, on-the-fly-generated pseudo-potentials, and the LDA exchange-correlation functional. For the structure with minimum energy (supercell a), the convergence criterion of 0.1 $\mathrm{kHz}$ for $C_{\mathrm{Q}}$ was met with a cut-off energy of $1.3 \mathrm{keV}$ and a $k$-point grid of $8 \times 8 \times 4$, which was adopted for the calculation of the $C_{\mathrm{Q}}$ of the other LTO unit cells. Modifications in the pseudopotentials (especially in the $\mathrm{Li}$ pseudo-potential) resulted in changes of up to $25 \mathrm{kHz}$ for the absolute value of $C_{\mathrm{Q}}$ in supercell a, whereas the replacement of the LDA exchange-correlation functional with PBE only yielded negligible changes of up to 0.5 $\mathrm{kHz}$.

\section{Results}

The X-ray diffractograms of the prepared samples (Fig. 2a) are consistent with literature. ${ }^{1722}$ While they do not allow
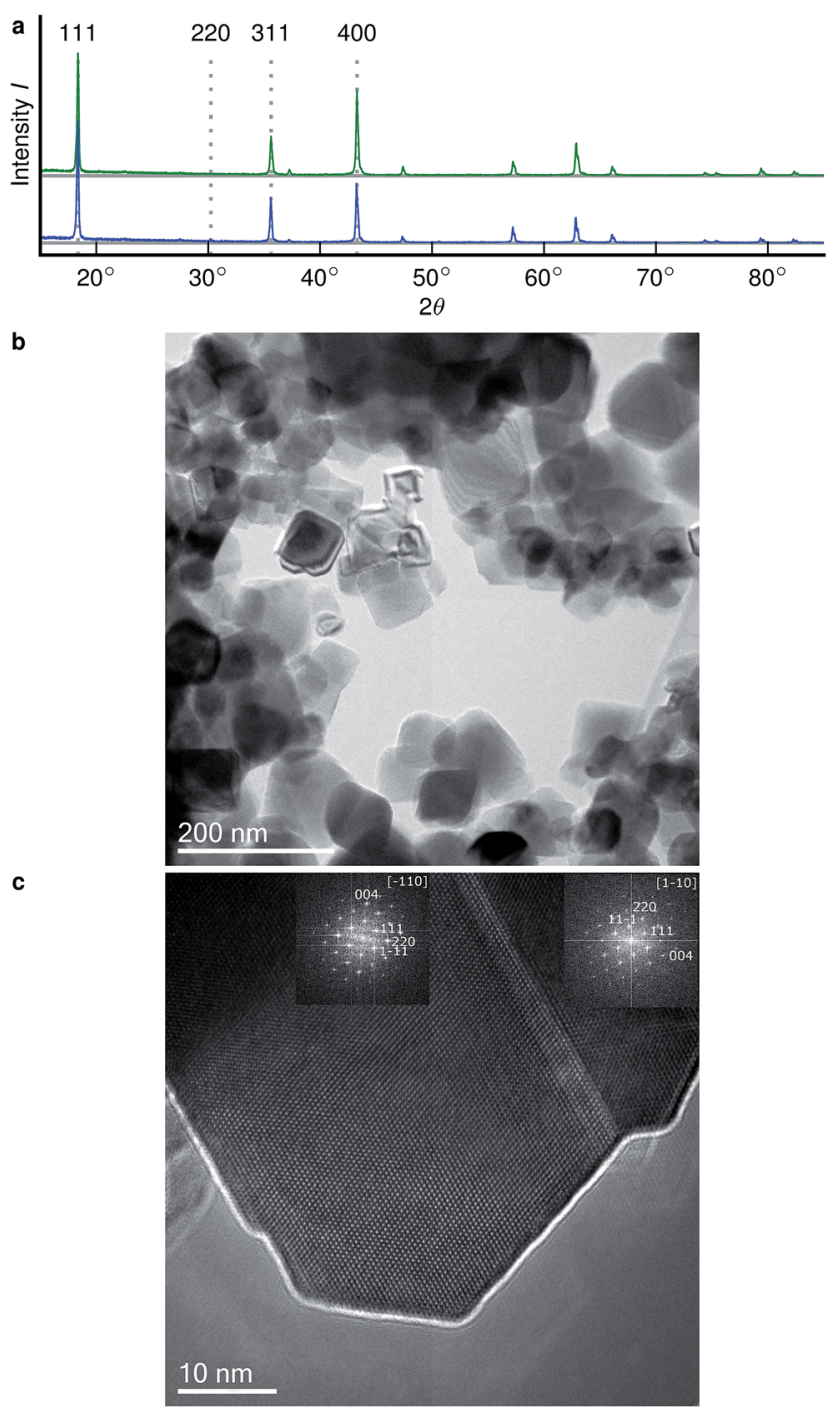

Fig. 2 (a) X-ray diffractograms of stoichiometric (blue) and overlithiated (green) LTO ( $x \approx 1.6$ ), scaled to the 111 reflection, with reflection positions (dotted lines) from simulations. (b) TEM image of overlithiated LTO, showing a grain size of less than $100 \mathrm{~nm}$. (c) HRTEM image of overlithiated LTO showing different crystallite orientations in one grain. 
a quantification of Li content, they are qualitatively in agreement with simulations as $I_{311} / I_{111}$ increases, $I_{400} / I_{111}$ decreases and $I_{220}$ almost disappears with overlithiation. The average grain sizes of the product as well as the educt are below $100 \mathrm{~nm}$ (Fig. 2b) with different crystallite orientations per grain (Fig. 2c).

Fig. 3 shows the spectrally resolved density of the SAE correlation time constant $\tau_{\mathrm{C}}$ at different temperatures. Individual spectra for selected $\tau_{\mathrm{C}}$ are shown in Fig. 4 .

\subsection{Stoichiometric LTO}

For stoichiometric LTO (Fig. 3a) the pattern of the spectrally resolved correlation time density is similar at all temperatures. However, a temperature increase does not lead to a simple shift of these patterns, as would be expected for dynamic processes following an Arrhenius law with a single $E_{\mathrm{a}}{ }^{12}$ Instead, fast processes are almost temperature independent, indicating a small $E_{\mathrm{a}}$, while temperature dependence is increasing for slower processes, suggesting a higher $E_{\mathrm{a}}$.

Signal components with a negative amplitude, which proved to be necessary for an accurate fit of the data, can be observed at $\tau_{\mathrm{C}} \approx 10 \mu \mathrm{s}$ and off-center in the spectral dimension on both sides of the main signal components along the whole $\tau_{\mathrm{C}}$ axis. The former might result from spin-spin $\left(T_{2}\right)$ relaxation effects, allowing unwanted coherences to contribute to the NMR signal. ${ }^{44}$ Hence the signal decay may contain some Gaussian contribution. Inverting a Gaussian function with an exponential kernel leads to signal contributions with negative amplitude. The origin of the latter component cannot be identified unequivocally. One possibility could be relaxation induced violations of coherence transfer selection rules, which could lead to such negative amplitudes on either side of a resonance. ${ }^{47}$

The change of the spectral features as a function of $\tau_{\mathrm{C}}$ is quite pronounced. Although broadened, the spectra display the pattern expected for quadrupolar interactions of a spin $3 / 2$ nucleus (Fig. 4a). With the exception of the fastest correlation times probed, the width of these features exceeds $\tau_{\mathrm{C}}{ }^{-1}$, hence it is unlikely that processes exhibiting a correlation time $\tau_{\mathrm{C}}$ are the cause for this line narrowing. We can thus assume that the width of the spectra represents the averaged magnitude of the EFG seen by the signal-inducing nuclei and that an estimate of the quadrupolar interaction is possible. Since no reference data of quadrupolar coupling constants of ${ }^{7} \mathrm{Li}$ ions in LTO are available, ab initio calculations were performed to estimate EFG values at lattice sites occupied by $\mathrm{Li}^{+}$. Because such a calculation needs to consider the stochastic occupancy of the $16 \mathrm{~d}$ sites in LTO even in the absence of defects, a set of supercells with systematic variation of $\mathrm{Li}$ and $\mathrm{Ti}$ on $16 \mathrm{~d}$ sites has been utilized (Fig. 1). Simulated $C_{\mathrm{Q}}$ values for the six different unit cells are summarized in Table 1.

\subsection{Overlithiated LTO}

A chemically overlithiated sample was used to study the impact of electronic defects, such as polaronic $\mathrm{Ti}^{3+}$ states, on the $\mathrm{Li}$ mobility in LTO. In Fig. $3 \mathrm{~b}$ the correlation between $\tau_{\mathrm{C}}$ and the static NMR spectrum is shown for overlithiated LTO. The general trends and patterns are similar to the stoichiometric LTO, but $\tau_{\mathrm{C}}$ of the dominant signal components are about two orders of magnitude shorter and the spectra are considerably broader. A quadrupolar origin of the line broadening is
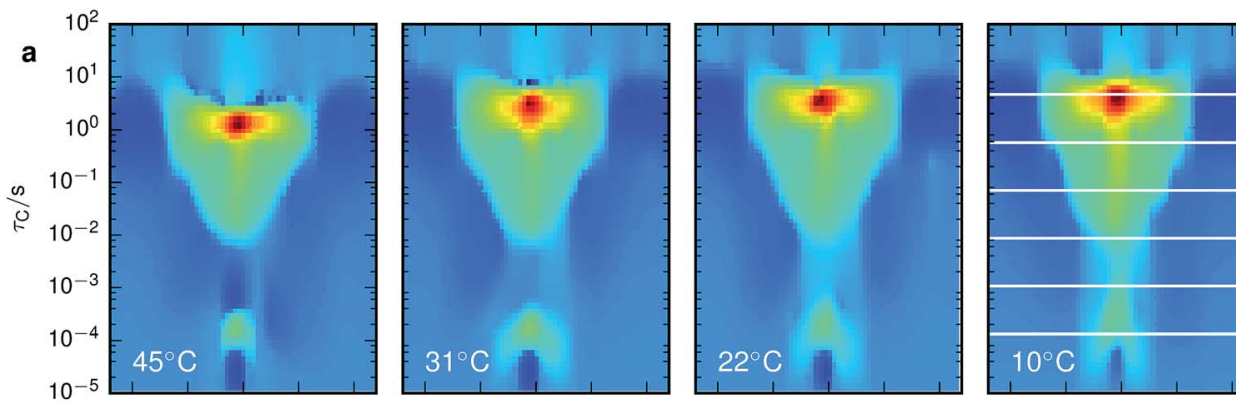

0.90
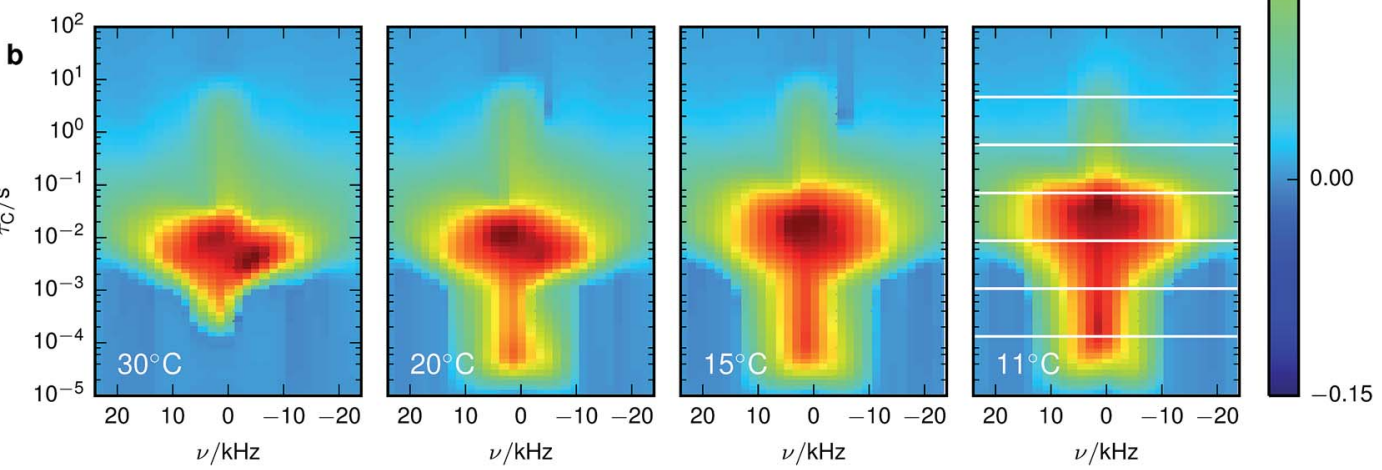

Fig. 3 Inversion of ${ }^{7} \mathrm{Li}$ SAE spectra recorded at different temperatures for (a) stoichiometric and (b) overlithiated LTO. The plots show the density of the correlation time $\tau_{C}$ from relatively fast processes (small $\tau_{C}$ ) to very slow processes (large $\tau_{C}$ ). The spectral dimension facilitates the individual NMR lineshape analysis of Li species with different mobilities. The white lines in the lowest temperature figures identify the slices shown in Fig. 4. 

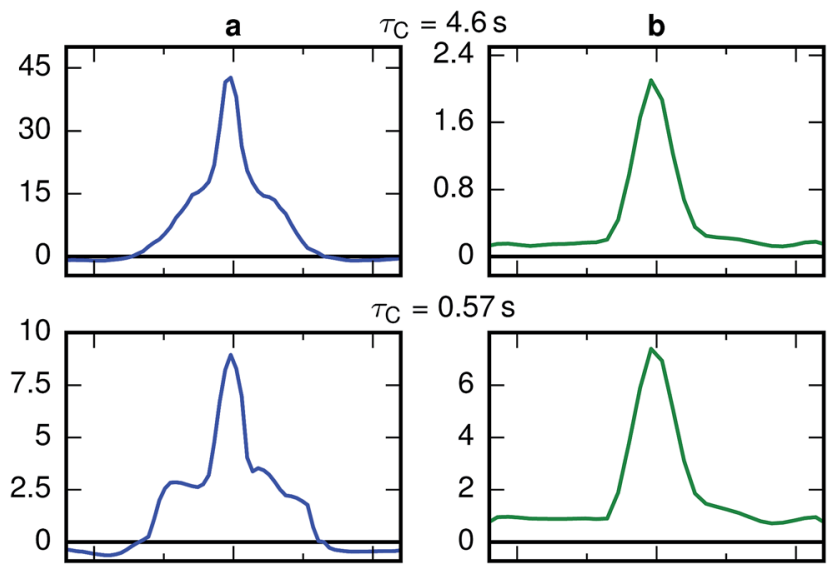

$=0.57 \mathrm{~s}$

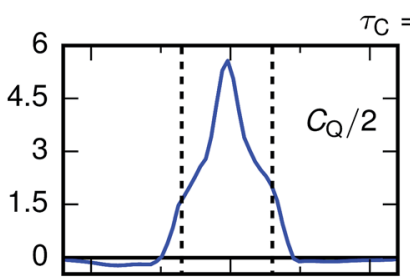

$\tau_{\mathrm{C}}=0.071 \mathrm{~s}$
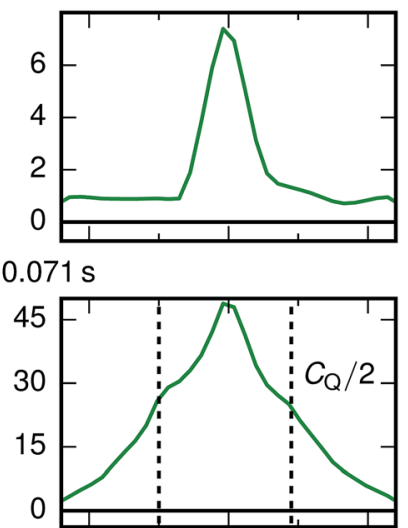

$\tau_{C}=0.0087 \mathrm{~s}$
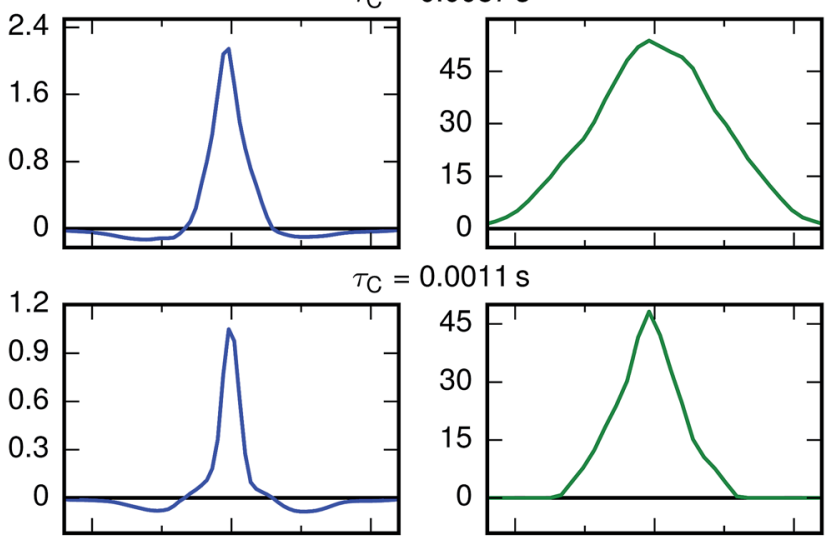

$=0.0011 \mathrm{~s}$

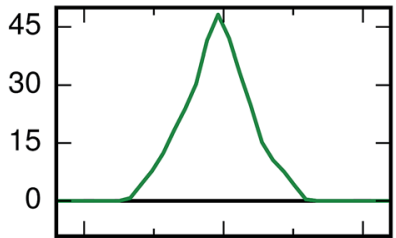

$\tau_{\mathrm{C}}=0.00013 \mathrm{~s}$
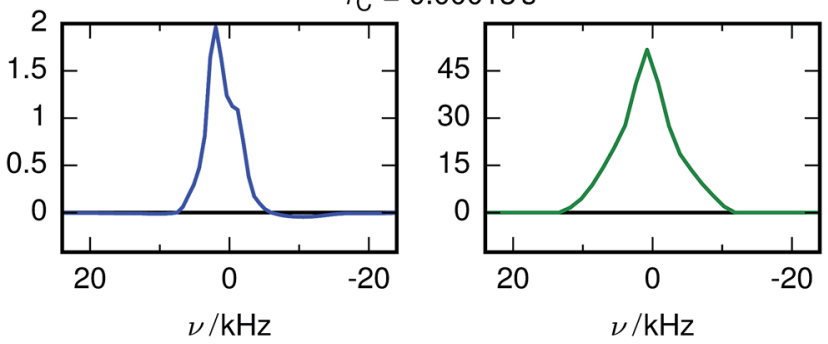

Fig. 4 Selected ${ }^{7}$ Li SAE spectra of (a) stoichiometric LTO at $10^{\circ} \mathrm{C}$ and (b) overlithiated LTO at $11{ }^{\circ} \mathrm{C}$. The spectra for $\tau_{\mathrm{C}}=0.071 \mathrm{~s}$ show how the quadrupolar coupling constant $C_{Q}$ can be extracted from the shoulders of a spin 3/2 powder NMR spectrum.

supported by the shape of the spectra, which again show - albeit additionally broadened - features as expected for static quadrupolar spectra of spin 3/2 nuclei (Fig. 4b).

It can be seen that a fraction of the overlithiated LTO shows a signal with similar $\tau_{\mathrm{C}}$ as the stoichiometric LTO. This is expected for two-phase formation upon overlithiation. ${ }^{\mathbf{1 4 - 1 6}}$ However, the signal fraction exhibiting such a reduced mobility in overlithiated LTO is smaller than the fraction of
Table 1 Summary of theoretically simulated quadrupolar coupling constants $\mathrm{C}_{\mathrm{Q}} / \mathrm{kHz}$ for the symmetry-inequivalent Li positions in six different $\mathrm{Li}_{8} \mathrm{Ti}_{10} \mathrm{O}_{24}$ supercells (Fig. $1 \mathrm{a}-\mathrm{f}$ ). The simulated values yield an order of magnitude estimate of the microscopic values for $\mathrm{C}_{\mathrm{Q}}$ of $\mathrm{Li}$ in LTO. The influence of different computational parameters introduces an additional uncertainty of up to $25 \mathrm{kHz}$

\begin{tabular}{llrrrrr}
\hline Li position & a & \multicolumn{1}{c}{$\mathrm{b}$} & \multicolumn{1}{c}{$\mathrm{c}$} & $\mathrm{d}$ & $\mathrm{e}$ & \multicolumn{1}{c}{$\mathrm{f}$} \\
\hline 8a & 61.3 & 62.7 & 14.4 & 46.6 & 285.4 & 280.6 \\
8a & 61.3 & 95.1 & 207.0 & 118.5 & 18.5 & 280.6 \\
8a & 16.2 & 95.1 & 204.8 & 118.7 & 288.1 & 14.6 \\
8a & 84.2 & 61.3 & 12.1 & 46.2 & 300.8 & 350.2 \\
8a & 83.7 & 160.3 & 238.8 & 146.9 & 32.0 & 350.6 \\
8a & 16.0 & 160.2 & 237.3 & 146.7 & 277.9 & 14.6 \\
16d & 34.1 & 71.5 & 36.2 & 54.1 & 99.2 & 145.0 \\
16d & 70.7 & 67.8 & 33.3 & 54.3 & 68.6 & 144.9 \\
& & & & & & \\
\hline
\end{tabular}

stoichiometric LTO if a strict two-phase separation according to $\mathrm{Li}_{4+x} \mathrm{Ti}_{5} \mathrm{O}_{12}=(1-x / 3) \mathrm{Li}_{4} \mathrm{Ti}_{5} \mathrm{O}_{12}+(x / 3) \mathrm{Li}_{7} \mathrm{Ti}_{5} \mathrm{O}_{12}$ is assumed. ${ }^{22}$ To distinguish different resonances, the spectrally resolved distribution of spin-lattice relaxation times $T_{1}$ of overlithiated LTO using an SR experiment with MAS was determined (Fig. 5). Two different spectral features are observed: a set of poorly resolved resonances with different $T_{1}$ at about $0 \mathrm{ppm}$ (regions $\mathrm{A}-\mathrm{C}$ ) and a broad line centered at about $-10 \mathrm{ppm}$ (region D). The frequencies of the former are overlapping with the resonances observed in stoichiometric LTO. ${ }^{19}$ The latter is due to the presence of paramagnetic $\mathrm{Ti}^{3+} \cdot{ }^{48}$ The spectral separation is likely caused by the formation of different phases rather than a solid solution upon chemical overlithiation. This is in agreement with microscopy results, ${ }^{15}$ where clear boundaries between overlithiated and stoichiometric phases were observed. Nonetheless, both signals show a short relaxation component for $100 \mathrm{~ms} \leq T_{1} \leqq 1 \mathrm{~s}$ (regions $\mathrm{B}$ and $\mathrm{D}$ ). This is considerably shorter than $T_{1}$ of stoichiometric LTO, whose projected distribution is shown in Fig. 5, and is indicative of Li ions in the vicinity of paramagnetic $\mathrm{Ti}^{3+}$.

The resonances at $0 \mathrm{ppm}$ show additional clearly distinguished relaxation components. The one at $T_{1} \approx 10 \mathrm{~s}$ (region A), which is only moderately reduced compared to $T_{1}$ of stoichiometric LTO, could be explained by some $\mathrm{Li}^{+}$exchange with overlithiated phases, yet considerably reduced compared to the above-mentioned component with fast $T_{1}$. Alternatively, such an increase could be caused by a variation of $\mathrm{Li}$ ion mobility, without the presence of paramagnetic centers in the region sampled by a Li ion. Either way, the reduction of $T_{1}$ shows that these phases are not macroscopically separated due to nonuniform sample overlithiation, which would cause a contribution with identical $T_{1}$ as in stoichiometric LTO. It also indicates that there is some heterogeneity in the distribution of mobility or size of the phases, otherwise a continuous distribution of $T_{1}$ would be expected. Finally, the component at $T_{1} \approx 20 \mathrm{~ms}$ (region C) is rather unusual. It does not show a considerable chemical shift, and it is in the immediate neighborhood of a peak with negative sign. It is likely that both peaks are caused by exchange, since we know from the SAE that corresponding mobility processes are taking place. 


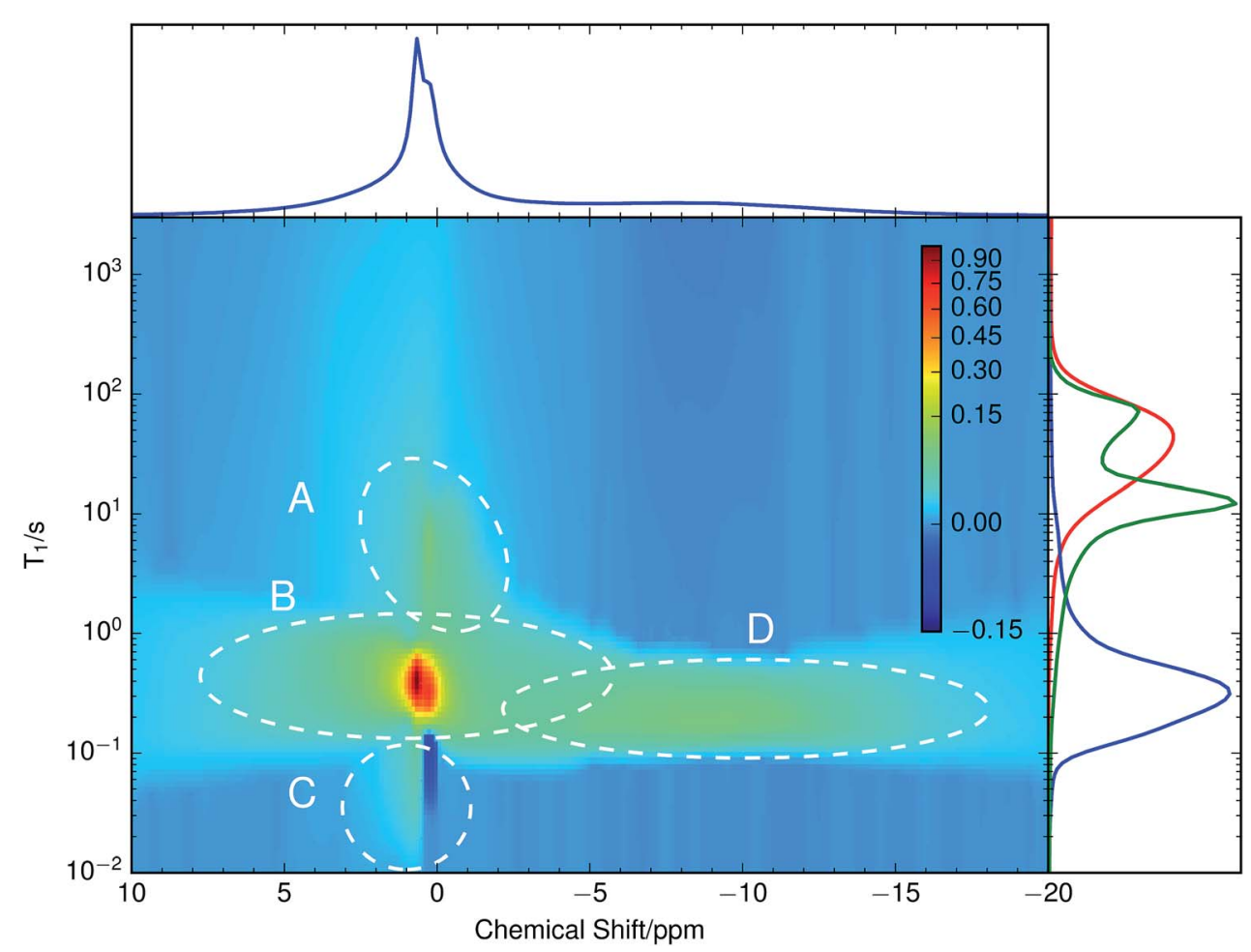

Fig. 5 Inversion of an ${ }^{7}$ Li MAS saturation recovery experiment on overlithiated LTO. The broad spectral feature at -10 ppm shows a completely different $T_{1}$ distribution to the rest of the spectrum. Overlaid with the sum projection of $T_{1}$ (blue) is the $T_{1}$ distribution of less overlithiated LTO (green) recorded in the same way and stoichiometric LTO recorded without MAS (red). Different regions are marked with dashed ellipses and labeled with capital letters for simpler identification.

Overlithiation in the limit of small $x$ was probed using a sample with $x \approx 0.2$. This sample does not show a feature around $-10 \mathrm{ppm}$ and the resonances around $0 \mathrm{ppm}$ are much narrower than for $x \approx 1.6$ and even slighty narrower than for $x=0$, consistent with data shown by Schmidt et al. ${ }^{13}$ Its $T_{1}$ distribution, shown in the side panel of Fig. 5 (green curve) consists of two clearly separated peaks at $T_{1} \approx 80 \mathrm{~s}$ and $T_{1} \approx$ $10 \mathrm{~s}$ as well as a long, low tail towards shorter relaxation times. The peak at longer $T_{1}$ coincides with the long $T_{1}$ edge of the stoichiometric LTO. The peak with shorter $T_{1}$ is somewhat reduced compared to the edge at short $T_{1}$ of the stoichiometric LTO, which would be consistent with a $T_{1}$ reduction due to accelerated motion, ${ }^{13}$ but not with the considerable relaxivity enhancement induced by paramagnetic $\mathrm{Ti}^{3+}$. This latter effect is likely responsible for the long, weak tail towards short $T_{1}$, which shows the onset of the features observed for the quickly relaxing signals (including the negative signal in region C) of the sample with $x \approx 1.6$.

\section{Discussion}

\subsection{Mobility modes of $\mathrm{Li}$ ions}

Experimentally observed $(\sim 10-40 \mathrm{kHz})$ and theoretically simulated $(\sim 20-300 \mathrm{kHz}) C_{\mathrm{Q}}$ values differ by a factor of between two and eight. Even though the first-principles simulated values show a rather large methodical error of up to $25 \mathrm{kHz}$, the differences cannot be attributed to this methodical error alone. In particular, the simulated values systematically overestimate experimental values. In contrast to the common way of interpretation, the experimentally observed $C_{\mathrm{Q}}$ might not result from microscopic, local EFGs on individual Li sites. Instead, consistent with molecular dynamics simulations, ${ }^{19}$ this suggests microscopic Li hopping between a limited number of sites on a much shorter timescale than the experimentally accessible timescale of $\sim 10^{-5} \mathrm{~s}$, causing a partial, anisotropic averaging of the individual EFGs. This results in an experimentally observed residual quadrupolar coupling, ${ }^{49-51}$ which is illustrated in Fig. 6 . Notice that at $\tau_{\mathrm{C}} \gtrsim 10 \mathrm{~s}$ the spectrum gets considerably broader, more consistent with the values obtained for $C_{\mathrm{Q}}$ from simulations. Considering the distribution of the spin-lattice relaxation time constant $T_{1}$ shown in the side panel of Fig. 5 , it is likely that this signal contribution with long $\tau_{\mathrm{C}}$ is caused by immobile $\mathrm{Li}$ ions that are relaxing with the relaxation time constant of quadrupolar order, $T_{1 \mathrm{Q}} \approx T_{1} / 3$, in an SAE experiment. ${ }^{28}$

Similar averaging effects, yet more pronounced due to the observed sharp quadrupolar pattern, have been found for fast ionic conductors. ${ }^{52}$ For LTO, this implies that to quantify ion mobility, two intrinsically different influences need to be considered - fast local mobility leading to the observation of residual quadrupolar coupling, and slower long-range mobility over larger distances between local domains, which gets probed by SAE NMR.

Since isotropic chemical shifts of Li in diamagnetic environments are distributed over a frequency range that is considerably narrower than typical values of $C_{\mathrm{Q}}$, such fast local mobility that causes a narrowing of quadrupolar patterns would 


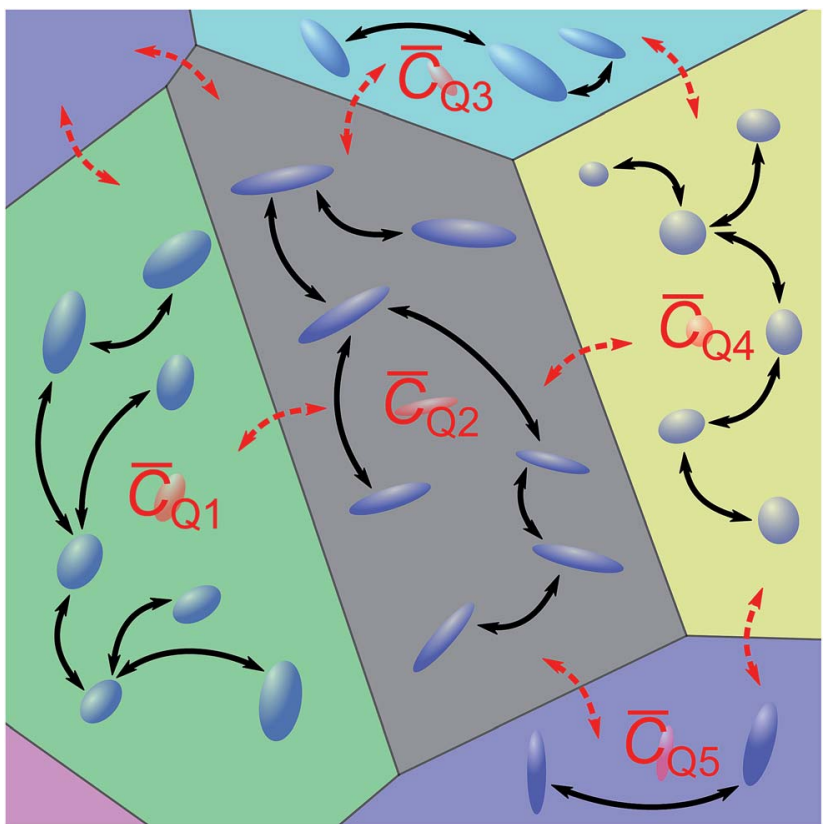

Fig. 6 Illustration of our suggested model of Li mobility. Li ions perform quick microscopic jumps (black solid arrows) between sites with different EFGs (blue ellipsoids) within a domain. Since these jumps are faster than the timescale probed by SAE NMR, the measured hopping rate describes movements between the domains (red dashed arrows) with different averaged residual EFGs (red ellipsoids).

also lead to an averaging of the isotropic chemical shift among the sites that form a local mobility domain in an experiment with MAS. Hence the conventional interpretation of isotropic ${ }^{6,7} \mathrm{Li}$ chemical shifts of LTO in terms of static Li occupancy of certain crystallographic sites ${ }^{20,22,53}$ could be incomplete or even unsuitable, and discrepancies of site populations between NMR and other techniques would be a consequence. ${ }^{19}$

\subsection{Incorporation of additional Li by overlithiation}

The observed differences in the SAE NMR spectrum between the stoichiometric and the overlithiated LTO sample (Fig. 3 and 4) arise from the presence of paramagnetic centers generated upon incorporation of additional $\mathrm{Li}^{+}$into LTO, which is chargecompensated primarily by reduction of diamagnetic $\mathrm{Ti}^{4+}$ to paramagnetic $\mathrm{Ti}^{3+}$. A high local concentration of $\mathrm{Ti}^{3+}$ ions causes a paramagnetic shift or a Knight shift and induces a considerable line broadening due to reduced $T_{2}$ relaxation time constants. ${ }^{48}$ Furthermore, both $\mathrm{Ti}^{3+}$ as well as the additionally incorporated $\mathrm{Li}^{+}$could change the EFG with a corresponding alteration of $C_{\mathrm{Q}}$. The increased mobility of Li ions in the overlithiated LTO, which is reflected by the reduced $\tau_{\mathrm{C}}$, can be linked directly with their vicinity to reduced $\mathrm{Ti}^{3+}$ centers. Notice that $\tau_{\mathrm{C}}$ for overlithiated LTO may also be affected by polaronic hopping of an electron between $\mathrm{Ti}$ ions rather than motion of the Li ion itself. Hence care must be taken when attempting a quantitative analysis of SAE experiments with potentially non-stationary paramagnetic centers.

A possible explanation for the increased average EFG probed by $C_{\mathrm{Q}}$ could be the presence of the additionally incorporated $\mathrm{Li}$ ions and a shorter average distance to the titanium due to a smaller repulsion caused by the reduced nominal Ti charge. This makes it easier for Li to approach Ti ions, thereby also speeding up ion transport. Alternatively, an increased average EFG may also be caused by smaller local domains, which would show poorer averaging.

A trend observed for stoichiometric as well as overlithiated LTO is the triangular shape of the spectrally resolved $\tau_{\mathrm{C}}$ pattern, which is narrowing towards shorter $\tau_{\mathrm{C}}$. Assuming that better averaging of the quadrupolar interaction is obtained for locally sampled domains with a larger number of sites, then this pattern would indicate that local mobility dispersed over more sites also causes faster motion between different domains. In other words, the more restricted the local mobility, the slower is the global mobility. An explanation could be that larger local domains also offer more anchors for jumps out of a domain. With more available jumping targets, i.e. Li vacancies or interstitial sites, the probability of a jump increases and $\tau_{\mathrm{C}}$ decreases, as observed. Alternatively, such an improved averaging could also be explained by poorer ordering within local domains, causing smaller residual quadrupolar couplings.

In the $T_{1}$ distribution shown in Fig. 5, region B is clearly affected by paramagnetic centers, which would contradict the two-phase model. A possible explanation could be densely entangled overlithiated and stoichiometric phases of small dimensions, which is consistent with data showing an exchange time constant of $\tau \approx 2 \mathrm{~ms}$ between the resonances. ${ }^{22}$ This is two to three orders of magnitude shorter than $T_{1}$ found in the distribution. Hence an equalization of $T_{1}$ is realistic for the majority of the two phases. An alternative interpretation could be a displacement of phase boundaries by simultaneous $\mathrm{Li}$ motion and polaronic electron hopping between neighboring $\mathrm{Ti}$ ions. However, such a model is incompatible with an observation of two-phase separation by microscopy techniques, ${ }^{15}$ which requires a much longer lifetime for phase boundaries to be detectable.

An explanation for the apparent discrepancy with results in the literature that suggest the formation of a solid solution upon overlithiation ${ }^{13}$ can be deduced from the $T_{1}$ experiment with the sample at $x \approx 0.2$. In this case only a very minor fraction of the $\mathrm{Li}$ is influenced by $\mathrm{Ti}^{3+}$, while a considerable fraction appears to show a slightly accelerated $T_{1}$ that would be consistent with an increased Li ion mobility. If charge of the additionally incorporated $\mathrm{Li}^{+}$ions is not compensated by reduction of $\mathrm{Ti}^{4+}$ to $\mathrm{Ti}^{3+}$, then it is conceivable that in the early stages of overlithiation the additional $\mathrm{Li}$ may be chargecompensated by removing oxygen atoms close to the grain surfaces. Since LTO is not an oxygen conductor at room temperature, initially a core-shell structure would be formed with the additional Li incorporated in the shell, possibly in the form of a solid solution. $\mathrm{Ti}^{4+}$ reduction would set in as soon as the surface regions of the LTO are oxygen depleted, leading to the two-phase configuration suggested above. From the overlap of $T_{1}$ of region A in Fig. 5 and the slightly accelerated $T_{1}$ component of the sample with $x \approx 0.2$, it seems possible that such a core-shell configuration, but with a considerably reduced shell volume, is maintained in the sample with $x \approx 1.6$. 
To quantitatively understand the evolution of the $T_{1}$ distribution, further experiments with a larger number of different overlithiation steps and a direct quantification of $\mathrm{Ti}^{3+}$ by electron paramagnetic resonance ${ }^{10}$ will be necessary.

\section{Conclusions}

A novel analysis technique for the inversion of solid-state NMR relaxation and SAE data was used to qualitatively study the mobility of $\mathrm{Li}$ ions in stoichiometric and chemically overlithiated LTO. Such an analysis facilitates, in analogy to established liquid-state NMR techniques, the separation of static or MAS NMR spectra into different components according to their $T_{1}$ or their $\tau_{\mathrm{C}}$. By employing an algorithm that does not depend on a non-negativity constraint, it is not necessary to assume that, for example, no exchange is taking place between different signal contributions. For the datasets shown in this work, the negative components proved to be necessary for a good fit to the experimental data. Although this method cannot be implemented as computationally efficient as the non-negativity constrained Tikhonov regularization, ${ }^{36}$ it is still possible to use it for two- and even three-dimensional inversion problems.

For LTO it was found that the ${ }^{7} \mathrm{Li}$ NMR spectra show a pronounced $\tau_{\mathrm{C}}$ dependence. From the discrepancy between experimentally observed quadrupolar couplings and numerical simulations it was concluded that fast local mobility of lithium ions between a limited number of sites is taking place and that the observed coupling constants represent residual quadrupolar couplings. Such a hypothesis also implies that resonances at different isotropic chemical shifts, as observed by MAS NMR experiments, may not be caused by static occupation of certain crystallographic sites. Furthermore, this means that SAE NMR is not suitable to directly quantify long-range ion motion in LTO as long as the size of the local domains is not known. Even NMR relaxation time measurements need to be assessed critically for their suitability for such a quantification, since multiple relaxation modes can be expected, which are potentially difficult to disentangle or for which it is not necessarily clear whether relaxation is dominated by long-range or short-range motion. However, effective parameters from NMR relaxation or SAE experiments may be well suited to compare differently synthesized samples of the same material or systematic material variations, for example by doping or variation of the stoichiometry.

Different contributions to the spectrally resolved SAE distribution show different temperature dependence, hence $\mathrm{Li}$ mobility cannot be described by a single activation energy or a small number of activation energies for jumps between particular crystallographic sites. Instead it is necessary to consider the stochastically occupied local environment of ions, as was predicted theoretically by DFT simulations. ${ }^{32}$ Our results indicate that it is not sufficient to simulate unit cells or small supercells with periodic boundary conditions accessible by DFT calculations, but that an intrinsically multiscalar simulation approach is required to cover local domains showing enhanced Li mobility as well as jumps between such domains.
For a more conclusive interpretation of the observed chemical shift values and time constant distributions, a refinement of simulations as well as additional spectroscopic and imaging experiments will be needed. In particular, atomistic as well as systematically coarse-grained simulations capable of capturing the essential dynamical processes of extended sample volumes appear promising, since the observed features in the relaxation time distribution indicate that relevant volume sizes even for the description of the two-phase behavior of overlithiated LTO could be within reach of current multiscale simulation methods. Preliminary results from simple kinetic Monte Carlo simulations with a single rate constant ${ }^{32}$ and randomized quadrupolar frequency distribution support the hypothesis of an averaging process on a timescale smaller than experimentally accessible (approx. $10^{-5} \mathrm{~s}$ ). Expanding the configurations (Fig. 1) sampled for quadrupolar frequencies by Li-16c defect structures results in a kinetically averaged $C_{\mathrm{Q}}$ much closer to experimental values.

\section{Acknowledgements}

We are grateful to Dr Peter Jakes and Steffen Kayser, M.Sc. of Forschungszentrum Jülich, Andreas Garhammer, B.Sc. and Hendrik Heenen, M.Sc. of Technische Universität München, and Dr Saskia Stegmaier of SUNCAT, SLAC for their technical assistance and fruitful discussions. Funding from the German Federal Ministry of Education and Research (BMBF project DESIREE, grant number 03SF0477A) is gratefully acknowledged.

\section{References}

1 M. V. Reddy, G. V. Subba Rao and B. V. R. Chowdari, Chem. Rev., 2013, 113, 5364-5457.

2 X. Sun, P. V. Radovanovic and B. Cui, New J. Chem., 2015, 39, 38-63.

3 Z. Yang, D. Choi, S. Kerisit, K. M. Rosso, D. Wang, J. Zhang, G. Graff and J. Liu, J. Power Sources, 2009, 192, 588-598.

4 C. Sandhya, B. John and C. Gouri, Ionics, 2014, 20, 601-620. 5 C. P. Grey and N. Dupré, Chem. Rev., 2004, 104, 4493-4512. 6 A. Deschanvres, B. Raveau and Z. Sekkal, Mater. Res. Bull., 1971, 6, 699-704.

7 Y.-B. He, M. Liu, Z.-D. Huang, B. Zhang, Y. Yu, B. Li, F. Kang and J.-K. Kim, J. Power Sources, 2013, 239, 269-276.

8 Z. Chen, I. Belharouak, Y.-K. Sun and K. Amine, Adv. Funct. Mater., 2012, 23, 959-969.

9 T. Ohzuku, A. Ueda and N. Yamamoto, J. Electrochem. Soc., 1995, 142, 1431-1435.

10 P. Jakes, J. Granwehr, H. Kungl and R.-A. Eichel, Z. Phys. Chem., 2015, 229, 1439-1450.

11 I. Leonidov, O. Leonidova, L. Perelyaeva, R. Samigullina, S. Kovyazina and M. Patrakeev, Phys. Solid State, 2003, 45, 2183-2188.

12 M. Wilkening, R. Amade, W. Iwaniak and P. Heitjans, Phys. Chem. Chem. Phys., 2007, 9, 1239.

13 W. Schmidt, P. Bottke, M. Sternad, P. Gollob, V. Hennige and M. Wilkening, Chem. Mater., 2015, 27, 1740-1750. 
14 S. Scharner, W. Weppner and P. Schmid-Beurmann, J. Electrochem. Soc., 1999, 146, 857-861.

15 M. Kitta, T. Akita, S. Tanaka and M. Kohyama, J. Power Sources, 2014, 257, 120-125.

16 D. Safronov, S. Novikova, A. Skundin and A. Yaroslavtsev, Inorg. Mater., 2012, 48, 57-61.

17 L. Aldon, P. Kubiak, M. Womes, J. Jumas, J. Olivier-Fourcade, J. Tirado, J. Corredor and C. Pérez Vicente, Chem. Mater., 2004, 16, 5721-5725.

18 J. Qiu, C. Lai, E. Gray, S. Li, S. Qiu, E. Strounina, C. Sun, H. Zhao and S. Zhang, J. Mater. Chem. A, 2014, 2, 6353-6358.

19 M. Vijayakumar, S. Kerisit, K. M. Rosso, S. D. Burton, J. A. Sears, Z. Yang, G. L. Graff, J. Liu and J. Hu, J. Power Sources, 2011, 196, 2211-2220.

20 P. Krtil, J. Dědeček, T. Kostlánová and J. Brus, Electrochem. Solid-State Lett., 2004, 7, A163-A166.

21 W. Schmidt and M. Wilkening, J. Phys. Chem. C, 2016, 120, 11372-11381.

22 H. Hain, M. Scheuermann, R. Heinzmann, L. Wünsche, H. Hahn and S. Indris, Solid State Nucl. Magn. Reson., 2012, 42, 9-16.

23 W. K. Pang, V. K. Peterson, N. Sharma, J.-J. Shiu and S.-h. Wu, Chem. Mater., 2014, 26, 2318-2326.

24 X. Lu, L. Gu, Y.-S. Hu, H.-C. Chiu, H. Li, G. P. Demopoulos and L. Chen, J. Am. Chem. Soc., 2015, 137, 1581-1586.

25 A. Laumann, H. Boysen, M. Bremholm, K. T. Fehr, M. Hoelzel and M. Holzapfel, Chem. Mater., 2011, 23, 2753-2759.

26 N. Takami, K. Hoshina and H. Inagaki, J. Electrochem. Soc., 2011, 158, A725-A730.

27 A. Kuhn, V. Epp, G. Schmidt, S. Narayanan, V. Thangadurai and M. Wilkening, J. Phys.: Condens. Matter, 2012, 24, 035901.

28 R. Böhmer, K. Jeffrey and M. Vogel, Prog. Nucl. Magn. Reson. Spectrosc., 2007, 50, 87-174.

29 Ģ. Vītinšs, G. Ķizāne, A. Lūsis and J. Tìiliks, J. Solid State Electrochem., 2002, 6, 311-319.

30 B. Ruprecht, M. Wilkening, R. Uecker and P. Heitjans, Phys. Chem. Chem. Phys., 2012, 14, 11974.

31 M. Vijayakumar, S. Kerisit, Z. Yang, G. L. Graff, J. Liu, J. A. Sears, S. D. Burton, K. M. Rosso and J. Hu, J. Phys. Chem. C, 2009, 113, 20108-20116.
32 B. Ziebarth, M. Klinsmann, T. Eckl and C. Elsässer, Phys. Rev. B: Condens. Matter Mater. Phys., 2014, 89, 174301.

33 G. Borgia, R. Brown and P. Fantazzini, J. Magn. Reson., 1998, 132, 65-77.

34 P. T. Callaghan, C. H. Arns, P. Galvosas, M. W. Hunter, Y. Qiao and K. E. Washburn, Magn. Reson. Imaging, 2007, 25, 441-444.

35 A. Lupulescu, M. Kotecha and L. Frydman, J. Am. Chem. Soc., 2003, 125, 3376-3383.

36 L. Venkataramanan, Y. Song and M. Hurlimann, IEEE Trans. Acoust., Speech, Signal Process., 2002, 50, 1017-1026.

37 G. Bodenhausen and R. R. Ernst, Mol. Phys., 1982, 47, 319328.

38 S. Rodts and D. Bytchenkoff, J. Magn. Reson., 2010, 205, 315318.

39 P. Fantazzini, F. Galassi, V. Bortolotti, R. Brown and F. Vittur, New J. Phys., 2011, 13, 065007.

40 Y.-Q. Song, G. Carneiro, L. M. Schwartz and D. L. Johnson, Phys. Rev. Lett., 2014, 113, 235503.

41 J. Granwehr and P. J. Roberts, J. Chem. Theory Comput., 2012, 8, 3473-3482.

42 W. Kraus and G. Nolze, J. Appl. Crystallogr., 1996, 29, 301303.

43 J. Jeener and P. Broekaert, Phys. Rev., 1967, 157, 232-240.

44 F. Qi, G. Diezemann, H. Böhm, J. Lambert and R. Böhmer, J. Magn. Reson., 2004, 169, 225-239.

45 M. Levitt, G. Bodenhausen and R. Ernst, J. Magn. Reson., 1984, 58, 462-472.

46 S. Clark, M. Segall, C. Pickard, P. Hasnip, M. Probert, K. Refson and M. Payne, Z. Kristallogr., 2005, 220, 567-570.

47 N. Müller, G. Bodenhausen and R. R. Ernst, J. Magn. Reson., 1987, 75, 297-334.

48 T. Kostlánová, J. Dědeček and P. Krtil, Electrochim. Acta, 2007, 52, 1847-1856.

49 M. Vogel and E. Rössler, J. Magn. Reson., 2000, 147, 43-58.

50 M. Vogel, C. Brinkmann, H. Eckert and A. Heuer, Solid State Nucl. Magn. Reson., 2002, 22, 344-362.

51 S. C. Shekar, J. A. Tang and A. Jerschow, Concepts Magn. Reson., Part A, 2010, 36, 362-387.

52 R. Bertermann and W. Müller-Warmuth, Z. Naturforsch., A: Phys. Sci., 1998, 53, 863-873.

53 M. Wagemaker, E. R. H. van Eck, A. P. M. Kentgens and F. M. Mulder, J. Phys. Chem. B, 2009, 113, 224-230. 